\title{
CHARACTERIZATIONS OF THE CONVERGENCE OF HARMONIC AVERAGES OF DOUBLE NUMERICAL SEQUENCES
}

\author{
ÁrpÁd FEKETE, IrINA GeORgIEVA ANd FERENC MórICZ
}

Abstract. In recent years, the almost sure central limit theorem has attracted widespread attention in Probability Theory. It involves the harmonic (also called logarithmic) averages of a certain numerical sequence formed from a sequence of independent, identically distributed random variables. The convergence behavior of the sequence of harmonic averages of a given numerical sequence was studied in [3] by the third author. Our main goal in this paper is to extend these characterization results from single to double numerical sequences of complex numbers.

Among others, the following Theorem $2 *$ is proved. Let $\left\{x_{i j}: i, j=1,2, \ldots\right\}$ be a double sequence of complex numbers. Necessary and sufficient condition for the existence of the bounded limit relation

$$
\mathrm{b}-\lim _{k, \ell \rightarrow \infty} \frac{1}{(\ln k)(\ln \ell)} \sum_{i=1}^{k} \sum_{j=1}^{\ell} \frac{x_{i j}}{i j}=\xi
$$

is that

$$
\mathrm{b}-\lim _{m, n \rightarrow \infty} \frac{1}{2^{m+n}} \max _{k \in J_{m}, \ell \in J_{n}}\left|\sum_{i=\mu_{m-1}+1}^{k} \sum_{j=\mu_{n-1}+1}^{\ell} \frac{x_{i j}-\xi}{i j}\right|=0,
$$

where

$$
J_{m}:=\left\{\mu_{m-1}+1, \mu_{m-1}+2, \ldots, \mu_{m}\right\}, \mu_{m}:=2^{2^{m}}, m=0,1, \ldots .
$$

Mathematics subject classification (2010): Primary 40B05, 40G99; Secondary 60F05, 60F15.

Keywords and phrases: Arithmetic averages, harmonic averages of double numerical sequences, bounded convergence in Pringsheim's sense, regular summability matrix, Toeplitz' theorem, bounded-regular summability matrix, Robison's theorem, law of large numbers, almost sure central limit theorem.

\section{REFERENCES}

[1] G. A. Bros amler, An almost everywhere central limit theorem, Math. Proc. Cambridge Philos. Soc., 104 (1988), 561-574.

[2] F. MóRICZ, On the harmonic averages of numerical sequences, Arch. Math. (Basel), 86 (2006), 375384.

[3] P. RÉvÉSZ, The Laws of Large Numbers, Academic Press, New York, 1968.

[4] G. M. Robison, Divergent double sequences and series, Trans. Amer. Math. Soc., 28 (1926), 50-73.

[5] A. Zygmund, Trigonometric Series, Vol. I, Cambridge Univ. Press, 1959. 